\title{
A critical review of exercise as a treatment for clinically depressed adults: time to get pragmatic
}

Schuch FB, Morres ID, Ekkekakis P, Rosenbaum S, Stubbs B. A critical review of exercise as a treatment for clinically depressed adults: time to get pragmatic.

Objective: Although considerable evidence supports the efficacy of exercise as an antidepressant treatment, critical reviews informing routine practice and future research directions are scarce.

Methods: We critically reviewed exercise studies for clinically depressed adults, focussing on the PICOS criteria referred to participants, interventions, comparisons, outcomes, and study designs.

Results: Most studies have not screened their samples for symptom heterogeneity. Also, they have employed heterogeneous exercise interventions and control groups that may lead to an underestimation of the benefits of exercise. In addition, pragmatic trials allowing scalable replication and implementation in routine practice are scarce. Future studies, can consider the research domain criteria as a diagnostic framework, and conduct moderator analyses to identify depressed subgroups with symptomatology and biopsychosocial characteristics associated with differential responses to exercise interventions. The search for biomarkers of the antidepressant responses to exercise should be prioritised. Further, non-physically active comparison groups should be used to minimise treatment cross-overs and thus the underestimation of the effects of exercise interventions. Finally, the use of outcome measures that maintain their validity at low and moderate levels of symptom severity and the development of trials with a pragmatic design are essential.

Conclusion: The current evidence base is fraught with methodological considerations that need to be taken into account in order to increase further our understanding on the impact of exercise as medicine in depression. Future research should include moderator analyses, incorporate biomarker assays, use appropriate control and comparison groups, assess outcomes with psychometrically sensitive measures, and prioritise pragmatic trials towards transition to routine practice.

\section{Felipe Barreto Schuch', loannis Dimitrios Morres', Panteleimon Ekkekakis ${ }^{3}$, Simon Rosenbaum ${ }^{4,5}$, Brendon Stubbs ${ }^{6,7}$}

'Post-Graduation Program of Medical Sciences: Psychiatry, Federal University of Rio Grande do Sul, Porto Alegre, Brazil; ${ }^{2}$ Exercise Psychology and Quality of Life Laboratory, Department of Physical Education and Sport Science, University of Thessaly, Trikala, Greece; ${ }^{3}$ Department of Kinesiology, lowa States University, Ames, IA, USA; ${ }^{4}$ School of Psychiatry, University of New South Wales, Sydney, Australia; Ingham Institute for Applied Medical Research, Liverpool, Australia; ${ }^{6}$ Physiotherapy Department, South London and Maudsley NHS Foundation Trust, Denmark Hill, London, UK; and ${ }^{7}$ Health Service and Population Research Department, Institute of Psychiatry, King's College London, De Crespigny Park, London, UK

Keywords: depression; exercise; physical activity; review; treatment

Felipe Barreto Schuch, Programa de Pós-Graduação em Ciências Médicas-Psiquiatria, Universidade Federal do Rio Grande do Sul, Porto Alegre, Brazil.

Tel: + 55513308 5624;

Fax: + 55513308 5624;

E-mail:felipe.schuch@ufrgs.br

Accepted for publication March 30, 2016

First published online 5 May 2016

\section{Significant outcomes}

- Future trials need to address various methodological and clinical considerations based on the 'participants, interventions, comparisons, outcomes, and study designs (PICOS)' criteria in order to advance further our understanding on the antidepressant impact of exercise for clinically depressed adults.

\section{Limitations}

- This is a selective critical review. Thus it is prone to bias as it ranks moderate in the evidence hierarchy. 


\section{Schuch et al.}

\section{Introduction}

A growing corpus of evidence provides substantial evidence that exercise is an efficacious treatment modality for mild to moderate depression (1-7). Previous high-profile reviews $(8,9)$, however, have cast doubt on the strength of the supporting evidence, highlighting methodological weaknesses in most of the reviewed randomised controlled trials (RCTs). For example, according to the latest edition of a Cochrane review (8), 'analysis of methodologically robust trials only shows a smaller effect in favour of exercise'. A recent critique of the methodological details of this review, however, raised serious doubts about this conclusion due, among other reasons, to problematic inclusion and exclusion criteria upon which trials were selected for the Cochrane review (10). It is, thus, conceivable that the antidepressant efficacy of exercise might have been underestimated $(5,10)$, doing a disservice to patients, and highlighting the central importance of sound methodological decisions in yielding accurate estimates of magnitude of the exercise efficacy as a treatment for depression (11).

In order to advance our understanding on the antidepressant impact of exercise and to help elucidate the reasons for the discrepant conclusions currently found in the literature, we conducted a selective critical review of relevant studies. For this purpose, we drew on the Preferred Reporting Items for Systematic Reviews and Meta-Analyses statement, focussing specifically on PICOS (12). Although the PICOS criteria can have a crucial impact on the outcome of any trial, critical assessments of these important factors are scarce on the existing literature of exercise and depression.

\section{Participants}

The use of participants with homogenous symptoms and similar changes in response to exercise would reduce within-group variance and increase the effect size associated with exercise interventions. Depression, however, is a heterogeneous disorder $(13,14)$ comprising diverse symptomatology (e.g. psychomotor retardation or agitation, increased or decreased appetite, insomnia or hypersomnia). Hence, patients with similar scores on measures of depression may experience dissimilar symptoms $(13,15,16)$. This heterogeneity in symptoms may reflect differences in underlying neurobiological processes $(13,15,16)$, suggesting that the same exercise prescription may be less effective for some patients and more effective for others. The heterogeneity of depression also involves various biopsychosocial factors. For example, a recent review provided initial evidence that clinical (severity of somatic symptoms), biological [brainderived neurotrophic factor, (BDNF) and tumour necrosis- $\alpha$ ], psychological (self-esteem and life satisfaction), and social factors (support and marital status) may moderate the antidepressant effects of exercise (17). Hence, developing a typology that would allow matching a depression 'type' to the most appropriate exercise prescription would both help advance research and facilitate clinical application.

The issue of classification has been the subject of a long, yet inconclusive debate (14) and the recent Diagnostic and Statistical Manual of the American Psychiatric Association (18) failed to provide new insights, especially from a neurobiological perspective (19). In light of this uncertainty, researchers may consider the Research Domain Criteria (RDoC) project. The $\mathrm{RDoC}$ aims to develop a taxonomy of mental disorders by taking advantage of research advances. To this end, the core of RDoC is a transdiagnostic matrix of functional dimensions, grouped on various domains including, among others, cognitive and reward-related systems. These are studied through seven units of analysis: (1) genes, (2) molecules, (3) cells, (4) neural circuits, (5) physiology, (6) behaviours and (7) selfreports (20). The adoption of the $\mathrm{RDoC}$ as a research framework is recommended by the National Institute of Mental Health (20), with clear implications for exercise and mental health research.

Research focussing on identifying patient's biomarkers and predictors of treatment response to exercise should be assigned high priority. This can be done by exploring moderators of the antidepressant effects of exercise, taking into account the following points (20). First, potential moderators must be chosen a priori, in a hypothesis-driven fashion, guided by theory or clinical experience. In this manner, the risk of facing 'data dredging' effects or spurious moderators is minimised. Second, data analysis should be based primarily on effect sizes instead of $p$ values, as a large sample size may generate 'statistically significant' results of little clinical relevance (21). Finally, antidepressant efficacy may be gauged by a battery of outcome measures, instead of a single measure, possibly enhancing reliability, reducing measurement error, and improving the odds of identifying important moderators (22). The reporting the mean changes in symptoms on the outcome measure will also help gauge clinical usefulness of interventions compared with controls groups.

\section{Interventions}

Identifying the optimal 'dose' of exercise in terms of frequency, intensity, duration, or overall volume is 
also crucial in developing effective exercise interventions for depression. Quantifying exercise in terms of energy expenditure is one possible way of defining 'dose'. For example, doses of 17.5 and $16.5 \mathrm{kcal} / \mathrm{kg} /$ week have been found to be effective as monotherapy and add-on therapy for patients with light-to-moderate (23) and severe $(24,25)$ depression, respectively. Interestingly, intensity in these trials was self-selected, possibly making exercise more suitable to patient preferences and needs.

The energy-expenditure approach to defining the exercise 'dose' may be consistent with public health recommendations for physical activity, but may not be the optimal method of deciphering potential biological mechanisms driving the antidepressant effects of exercise (26). Exercise prescriptions designed to specifically target putative mechanisms of the antidepressant effects of exercise could potentially achieve even greater benefits. A prescribed amount of exercise could be achieved via different combinations of frequency, duration, and intensity. However, each of these may be related to different mechanisms underlying the antidepressant effects of exercise, possibly resulting in a varying degree of symptom reduction. Some patients may reach the same prescribed amount of exercise via low-intensity and long-duration interventions (e.g. walking for a long distance) or via high intensity for a short duration (e.g. brief periods of running). Given the multitude of possible ways of reaching the same total amount of energy expenditure, identifying the frequency, duration, and intensity that optimally stimulate the biological mechanisms underlying the antidepressant effects of exercise becomes difficult, if not impossible. Instead, dose-response studies that manipulate specific parameters of the exercise dose (i.e. frequency, duration, intensity) specifically targeted for each individual, ideally in a factorial design, should be preferred, despite their high cost.

Gaining better understanding of the neurobiological processes underlying the antidepressant effects of exercise (26) will be crucial in designing more effective exercise interventions. For this purpose, hormonal, oxidative-stress, and neurogenetic pathways should be considered (26). The upregulation of BDNF (27) may warrant particular attention. BDNF promotes the process of neurogenesis. Acute bouts of exercise have been found to increase the serum levels of BDNF in psychiatric patients (26 28-32). Both exercise intensity and volume play a significant role in the magnitude of the exercise-induced effect on BDNF (33). It should be noted, however, that the BDNF response to long-term exercise in people with major depression is presently less clear (26,34-36).

\section{Comparisons}

Similar to other non-pharmacological treatments $(37,38)$, it has not been possible to disentangle the relative contribution of the effect of exercise on depression versus other non-exercise-specific factors, which can also play a role. For example, in most cases, exercising involves social interaction, which may have its own antidepressant effects, independent of exercise (39). However, social support has not been shown to be a strong predictor of subsequent depression (40) or contributor to the antidepressant effects of exercise (41). Nevertheless, trial arms must fully balance social influences to rule out any confounding impact on between-group comparisons and safeguard internal validity.

A common trend in RCTs investigating the antidepressant effects of exercise is to employ 'control' groups that also receive exercise, albeit of a different modality and/or intensity than that administered to the 'treatment' groups (e.g. aerobic exercise is compared with strength and flexibility training). The rationale typically offered for employing such groups as controls is that the current literature does not contain descriptions of specific mechanistic pathways by which these alternate exercise modalities might influence depression. Rather than being inert, however, alternate modalities of exercise have consistently been associated with statistically reliable and clinically meaningful reductions in depressive symptoms (42). As all modalities of exercise share many common biological features (e.g. repeated cycles of muscular contraction and relaxation, some degree of stimulation of the cardiovascular system), the employment of comparators also engaged in a form of exercise as 'control' may introduce a critical confound in exercise studies for depression.

As one example, based on the results of an RCT comparing aerobic exercise, strength exercise, and a 'control' arm engaged in 'relaxation,' the authors stated: 'Our findings do not support a biologically mediated effect of exercise on symptom severity in depressed patients' (43). This conclusion was intended to reflect the lack of significant differences in post-intervention depression scores between the three groups. All groups, however, including the 'control' group that was described as engaged in 'relaxation,' exhibited meaningful improvements in physical fitness (aerobic capacity and strength) and, accordingly, also showed substantially reduced post-intervention depressive symptomatology. Specifically, from pre- to post-intervention, the aerobic exercise group exhibited a standardised mean difference (SMD) of -1.15 (95\% CI $-1.56-0.75$ ), the strength intervention showed a SMD of -1.57 


\section{Schuch et al.}

(95\% CI -2.00 to -1.14 ), and the 'relaxation' group had a SMD of -1.27 (95\% CI -1.68 to -0.86 ). Although the provision of a fitness-enhancing exercise intervention to the group designated as 'control' arguably can turn the aforementioned conclusion from this trial on its head, this crucial confound only received a brief and rather inconspicuous acknowledgement in the discussion: 'Limitations include confounding due to a possible antidepressant effect of the intervention used in our control group (relaxation training)' (39).

In another RCT that compared a group engaged in aerobic exercise with a 'control' group engaged in a treatment described as 'stretching exercise,' the researchers concluded: 'our trial data do not support any effect of aerobic exercise on depressive symptoms' (44). In actuality, however, besides 20 min of stretching, participants in the 'control' group also engaged in $25 \mathrm{~min}$ of low-intensity aerobic exercise, including $10 \mathrm{~min}$ of 'warm-up on a stationary bike' and $15 \mathrm{~min}$ of 'throwing and catching balls.' As in the previous trial, it is noteworthy to mention that both groups exhibited large reductions in depressive symptoms (aerobic, SMD $-1.37,95 \%$ CI -1.78 to -0.96 ; stretching, SMD -1.51 , $95 \%$ CI -1.92 to -1.10) mirroring an entirely different-(positive) antidepressant status for exercise compared with the one presented by the trialists.

To avoid misleading conclusions, trialists, as well as authors, reviewers, editors, and readers engaged in critical appraisal, should consider the magnitude of changes in depressive symptoms in both the experimental and control groups, especially when the control groups also engaged in a type of exercise. The necessity of scrutinising the nature of treatments provided to groups described as 'controls' is also underscored by a recent meta-analysis of exercise trials for depression (42). This study has found uncommonly large reductions in depressive symptoms in 'control' groups receiving alternate exercise treatments (e.g. stretching) (42). These reductions were two times larger when compared with those reductions experienced by control groups assigned to trials on antidepressant medication.

\section{Outcomes}

Widely used outcome measures, such as the clinician-administered Hamilton Rating Scale for depression (45), have been known to present various psychometric problems, including lack of unidimensionality and poor ability to detect changes among individuals with mild to moderate depressive symptoms $(46,47)$. These problems may be particularly pertinent to studies investigating the antidepressant effects of exercise because exercise is a treatment that is specifically recommended for individuals with mild to moderate symptom severity. In the latest edition of the Cochrane review on exercise for depression (8), $>90 \%$ of the reviewed trials included patients with mild to moderate levels of depressive symptoms. Hence, the use of the Hamilton rating scale may not accurately reflect the magnitude of the antidepressant effect of exercise. Instead, recently developed scales based on Diagnostic and Statistical Manual of Mental Disorders, fourth edition (DSM-IV) criteria, such as the Inventory of Depressive Symptoms, may more accurately reflect changes across all levels of symptom severity (48). Thus, their use in future trials is strongly recommended.

\section{Study design}

RCTs are widely considered the 'gold standard' for establishing the causal effect of a given therapy on a certain clinical outcome. Establishing the causal effect, however, is conditional upon the degree of bias inherent in the experimental design or the extent to which the trials meet important criteria of methodological quality $(49,50)$. Given that risk of bias has been shown to be inversely associated with the magnitude of intervention effects (51), trials with a methodologically robust design are essential. Methodological criteria commonly considered hallmarks of a robust design include the generation of truly random allocation sequences, the successful concealment of group allocation, the blinding of outcome assessors, intention-to-treat analyses, the complete reporting of point estimates and variability indices on the primary outcome measures, and between-group baseline balance in the most important prognostic indicators including the primary outcome. Blinding participants and treatment providers are also considered crucial methodological criteria. However, the nature of an exercise intervention makes it impossible to blind both the participants themselves and the individuals administering the exercise interventions, clearly this is a particular challenge in control group arms of trials. Collecting and reporting data on drop-out rates, side effects, other adverse events, and the number needed to treat can also offer valuable insight into the acceptability of exercise as an intervention strategy. Finally, follow-up assessments can provide muchneeded information about the enduring effects of exercise interventions.

Over the past 30 years, the methodological quality of RCTs investigating the antidepressant effects of exercise has improved, reflected by improved adherence with reporting standards (52-54). Recent RCTs, in particular, tend to be of high methodological 
quality by commonly employed criteria $(25,55)$ and sample sizes have been substantially increased (49). Pragmatic RCTs, however, are scarce (41). Pragmatic RCTs are characterised by high external validity (outcome generalisability) by virtue of methodological features that are more closely aligned with 'real life' practice norms, such as interventions delivered in routine practice and inclusive (nonrestrictive) eligibility criteria for participation (56). Thus, pragmatic RCTs can be invaluable in facilitating the translation of clinical trial results to routine practice. This is of major importance given that practitioners are faced with a series of challenges in treating depressed patients. These challenges include primarily limited time and resources. Thus, we encourage researchers to conduct pragmatic RCTs. Given that a number of clinicians report lack of confidence in designing appropriate exercise prescriptions as a result of the aforementioned challenges, and that supervised trials present a lower drop-out rate (57), it is noteworthy that a pragmatic trial involving supervised-based exercise of preferred (self-selected) intensity yielded promising results in terms of safety, compliance, and depressive symptom reductions (41).

\section{Conclusion}

Specifying and advancing the current understanding of the antidepressant effects of exercise in relationship to the PICOS criteria is an important clinical topic with broad implications. The factors associated with the PICOS criteria that were reviewed here should be considered not only when designing future trials aiming to advance further previous trial outcomes but also when critically appraising published RCTs. In particular, with reference to participants, researchers should consider the RDoC (not only the DSM) as a diagnostic framework and include moderator analyses. Regarding the design of exercise interventions, the relationships between potential biological mediators (e.g. BDNF) and components of the exercise dose (e.g. volume or intensity) should be considered. With regard to control or comparison groups, while balancing nonspecific effects (e.g. social interaction) is essential in safeguarding internal validity, the use of alternatemodality exercise interventions as 'controls' creates serious confounds and should be avoided especially as alternate exercise modalities bring about antidepressant effects. In evaluating the results of trials employing exercising groups as 'controls,' trialists and critical readers are urged to assess the clinical meaningfulness of pre-to-post changes in depressive symptoms within these groups in addition to between-group comparisons post-intervention.
With respect to outcomes, psychometric scales that can accurately reflect changes at mild and moderate levels of depressive symptomatology should be preferred, as these are likely to be the most prevalent levels of symptom severity in recruited samples. Lastly, concerning study designs, researchers are encouraged to conduct more pragmatic (effectiveness) RCTs, to pave the way for the development of more practical and scalable exercise interventions that will be embedded in routine practice.

\section{Acknowledgements}

The authors would like to thank the Conselho nacional desenvolvimento científico e tecnológico (CNPq), Coordenação de aperfeiçoamento de pessoal de nível superior (CAPES), and the Exercise Psychology and Quality of Life Laboratory, Department of Physical Education and Sport Science, University of Thessaly, Trikala, Greece. Authors' Contribution: F.B.S., I.D.M., P.E., S.R., and B.S. contributed to the manuscript design and writing. All authors agreed with the final version of the manuscript.

\section{Financial Support}

The authors received no specific funding for this review from any agency, commercial entity, or notfor-profit organisation.

\section{Conflicts of Interest}

The authors declare no potential conflict of interest.

\section{References}

1. National Instituce for Clinical Excellence (NICE). Depression: the treatment and management of depression in adults, 2009. www.nice.org.uk/CG90

2. Anderson IM, Ferrier IN, Baldwin RC et al. Evidencebased guidelines for treating depressive disorders with antidepressants: a revision of the 2000 British Association for Psychopharmacology guidelines. J Psychopharmacol 2008;22:343-396.

3. Cleare A, Pariante C, Young A et al. Evidence-based guidelines for treating depressive disorders with antidepressants: a revision of the 2008 British Association for Psychopharmacology guidelines. J Psychopharmacol 2015;29:459-525.

4. Malhi GS, Bassett D, Boyce P et al. Royal Australian and New Zealand College of Psychiatrists clinical practice guidelines for mood disorders. Aust N Z J Psychiatry 2015;49:1087-1206.

5. Schuch FB, Vancampfort D, Richards J, Rosenbaum S, $\mathrm{W}_{\text {ARD }}$ PB, STUBBS B. Exercise as a treatment for depression: a meta-analysis adjusting for publication bias. J Psychiatr Res 2016;77:42-51. 
6. Rethorst CD, Wipfli BM, LANDERs DM. The antidepressive effects of exercise: a meta-analysis of randomized trials. Sports Med 2009;39:491-511.

7. Craft LL, LANDERS DM. The effects of exercise on clinical depression and depression resulting from mental illness: a meta-regression analysis. J Sport Exerc Psychol 1998;20: 339-357.

8. Cooney GM, Dwan K, Greig CA et al. Exercise for depression. Cochrane Database Syst Rev 2013;9:CD004366.

9. LAWLOR DA, HOPKER SW. The effectiveness of exercise as an intervention in the management of depression: systematic review and meta-regression analysis of randomised controlled trials. BMJ 2001;322:763-767.

10. ЕккекакіS P. Honey, I shrunk the pooled SMD! Guide to critical appraisal of systematic reviews and meta-analyses using the Cochrane review on exercise for depression as example. Ment Health Phys Act 2015;8:21-36.

11. Schuch FB, De Almeida Fleck MP. Is exercise an efficacious treatment for depression? A comment upon recent negative findings. Front Psychiatry 2013;4:20.

12. Liberati A, Altman DG, Tetzlaff J et al. The PRISMA statement for reporting systematic reviews and meta-analyses of studies that evaluate health care interventions: explanation and elaboration. Ann Intern Med 2009;151:W-65-W-94.

13. Parker G. Beyond major depression. Psychol Med 2005;35:467-474.

14. Fried EI, Nesse RM. Depression is not a consistent syndrome: an investigation of unique symptom patterns in the STAR*D study. J Affect Disord 2015;172:96-102.

15. Spanemberg L, Caldieraro MA, Vares EA et al. Biological differences between melancholic and nonmelancholic depression subtyped by the CORE measure. Neuropsychiatr Dis Treat 2014;10:1523-1531.

16. Caldieraro MA, Baeza Fl, Pinheiro DO, Ribeiro Mr, PARKER G, FLECK MP. Clinical differences between melancholic and nonmelancholic depression as defined by the CORE system. Compr Psychiatry 2013;54:11-15.

17. Schuch FB, Dunn AL, Kanitz AC, Delevatti RS, FLECK MP. Moderators of response in exercise treatment for depression: a systematic review. J Affect Disord 2016;195:40-49.

18. American Psychiatric Association. Diagnostic and statistical manual of mental disorders, 5th edn. Arlington, TX: American Psychiatric Association, 2013.

19. Nemeroff CB. The burden of severe depression: a review of diagnostic challenges and treatment alternatives. J Psychiatr Res 2007;41:189-206.

20. Insel T, Cuthbert B, Garvey $M$ et al. Research domain criteria (RDoC): toward a new classification framework for research on mental disorders. Am J Psychiatry 2010;167: 748-751.

21. Kraemer hC, Wilson GT, Fairburn CG, Agras WS. Mediators and moderators of treatment effects in randomized clinical trials. Arch Gen Psychiatry 2002;59: 877-883.

22. Wallace ML, Frank E, Kraemer HC. A novel approach for developing and interpreting treatment moderator profiles in randomized clinical trials. JAMA Psychiatry 2013;70: 1241-1247.

23. Dunn Al, Trivedi MH, Kampert JB, Clark CG, Chambliss HO. Exercise treatment for depression: efficacy and dose response. Am J Prev Med 2005;28:1-8.
24. Schuch FB, Vasconcelos-Moreno MP, Borowsky C, FLECK MP. Exercise and severe depression: preliminary results of an add-on study. J Affect Disord 2011;133:615-618.

25. Schuch FB, Vasconcelos-Moreno MP, Borowsky C, Zimmermann AB, Rocha NS, Fleck MP. Exercise and severe major depression: effect on symptom severity and quality of life at discharge in an inpatient cohort. J Psychiatr Res 2015;61:25-32.

26. Schuch FB, Deslandes AC, Stubbs B, Gosmann NP, Silva CT, FLECK MP. Neurobiological effects of exercise on major depressive disorder: a systematic review. Neurosci Biobehav Rev 2016;61:1-11.

27. Deslandes A, Moraes H, Ferreira $C$ et al. Exercise and mental health: many reasons to move. Neuropsychobiology 2009;59:191-198.

28. Broocks A, Ahrendt U, Sommer M. Physical training in the treatment of depressive disorders. Psychiatr Prax 2007;34 (Suppl.):S300-S304.

29. Heyman E, Gamelin FX, Goekint M et al. Intense exercise increases circulating endocannabinoid and BDNF levels in humans-possible implications for reward and depression. Psychoneuroendocrinology 2012;37:844-851.

30. Huang T, Larsen KT, Ried-Larsen M, Moller NC, ANDERSEN LB. The effects of physical activity and exercise on brain-derived neurotrophic factor in healthy humans: a review. Scand J Med Sci Sports 2014;24:1-10.

31. Szuhany KL, Bugatti M, Отto MW. A meta-analytic review of the effects of exercise on brain-derived neurotrophic factor. J Psychiatr Res 2015;60:56-64.

32. Schuch FB, da Silveira LE, de Zeni TC et al. Effects of a single bout of maximal aerobic exercise on BDNF in bipolar disorder: a gender-based response. Psychiatry Res 2015;229:57-62.

33. Schmolesky MT, Webi DL, Hansen RA. The effects of aerobic exercise intensity and duration on levels of brainderived neurotrophic factor in healthy men. J Sports Sci Med 2013;12:502-511.

34. Schuch FB, Vasconcelos-Moreno MP, Borowsky C et al. The effects of exercise on oxidative stress (TBARS) and BDNF in severely depressed inpatients. European Archives of Psychiatry and Clinical Neuroscience 2014;264:605-613.

35. Salehi I, Hosseini SM, Haghighi M et al. Electroconvulsive therapy and aerobic exercise training increased BDNF and ameliorated depressive symptoms in patients suffering from treatment-resistant major depressive disorder. J Psychiatr Res 2014;57:117-124.

36. Toups MSP, Greer TL, Kurian BT et al. Effects of serum brain derived neurotrophic factor on exercise augmentation treatment of depression. J Psychiatr Res 2011;45:1301-1306.

37. Donovan HS, Kwekкeboom KL, Rosenzweig MQ, Ward SE. Non-specific effects in psycho-educational intervention research. West J Nurs Res 2009;31:983-998.

38. Weimer K, Colloca L, Enck P. Placebo effects in psychiatry: mediators and moderators. Lancet Psychiatry 2:246-257.

39. Stathopoulou G, Powers MB, Berry aC, Smits JaJ, Отто MW. Exercise interventions for mental health: a quantitative and qualitative review. Clin Psychol Sci Prac 2006;13:179-193.

40. Morres I, Van de Vliet P, Knapen J, VanCoppenolle H. Determinants of exercise participation in relationship to severity of clinical depression in adult female psychiatric in-patients. Eur Bull Adapt Phys Act 2003;2. 


\section{A critical review of exercise as a treatment for clinically depressed adults}

41. Callaghan P, Khalil E, Morres I, Carter T. Pragmatic randomised controlled trial of preferred intensity exercise in women living with depression. BMC Public Health 2011;11:465.

42. Stubbs B, Vancampfort D, Rosenbaum $\mathrm{S}$ et al. Challenges establishing the efficacy of exercise as an antidepressant treatment: a systematic review and meta-analysis of control group responses in exercise randomised controlled trials. Sports Med 2015;1-15 (in press).

43. Krogh J, Saltin B, Gluud C, Nordentoft M. The DEMO trial: a randomized, parallel-group, observer-blinded clinical trial of strength versus aerobic versus relaxation training for patients with mild to moderate depression. J Clin Psychiatry 2009;70:790-800.

44. Krogh J, Videbech P, Thomsen C, Gluud C, Nordentoft M. DEMO-II trial. Aerobic exercise versus stretching exercise in patients with major depression-a randomised clinical trial. PLoS One 2012;7:e48316.

45. Hamilton M. Development of a rating scale for primary depressive illness. Br J Soc Clin Psychol 1967;6:278-296.

46. IsACSSON G, AdLER M. Randomized clinical trials underestimate the efficacy of antidepressants in less severe depression. Acta Psychiatr Scand 2012;125:453-459.

47. Salum GA, Manfro GG, Fleck MP. What is not 'effective' in mild to moderate depression: antidepressants or the Hamilton Rating Scale for depression? CNS Spectr 2011;16:99.

48. Helmreich I, Wagner S, Mergl $\mathrm{R}$ et al. Sensitivity to changes during antidepressant treatment: a comparison of unidimensional subscales of the Inventory of Depressive Symptomatology (IDS-C) and the Hamilton Depression Rating Scale (HAMD) in patients with mild major, minor or subsyndromal depression. Eur Arch Psychiatry Clin Neurosci 2012;262:291-304.
49. АковеNG A. Understanding randomised controlled trials. Arch Dis Child 2005;90:840-844.

50. Moher D, Schulz KF, Altman DG, Group C. The CONSORT statement: revised recommendations for improving the quality of reports of parallel-group randomised trials. Lancet 2001;357:1191-1194.

51. Wood L, EgGer M, Gluud LL et al. Empirical evidence of bias in treatment effect estimates in controlled trials with different interventions and outcomes: metaepidemiological study. BMJ 2008;336:601-605.

52. Perraton LG, Kumar S, Machotka Z. Exercise parameters in the treatment of clinical depression: a systematic review of randomized controlled trials. J Eval Clin Pract 2010;16: 597-604.

53. Stanton R, Reaburn P. Exercise and the treatment of depression: a review of the exercise program variables. J Sci Med Sport 2013;2:177-182.

54. Morres I, Stathi A, Martinsen EW, Sørensen M. Physical exercise and major depressive disorder in adult patients. In: HD Papaioannou AG, editor Routledge companion to sport and exercise psychology: global perspectives and fundamental concepts. UK: Taylor \& Francis, 2014; p. $823-834$.

55. Hallgren M, Kraepelien M, Öjehagen A et al. Physical exercise and internet-based cognitive behavioural therapy in the treatment of depression: randomised controlled trial. Br J Psychiatry 2015;207:227-234.

56. Ноторғ M. The pragmatic randomised controlled trial. Adv Psychiatr Treat 2002;8:326-333.

57. Stubbs B, Vancampfort D, Rosenbaum $S$ et al. Dropout from exercise randomized controlled trials among people with depression: a meta-analysis and meta regression. J Affect Disord 2016;190:457-466. 